\title{
Ion-implanted compliant and patternable electrodes for miniaturized dielectric elastomer actuators
}

\author{
Samuel Rosset; Muhamed Niklaus; Philippe Dubois; Herbert R. Shea
}

\section{Copyright 2008 Society of Photo-Optical Instrumentation Engineers.}

This paper was published in Proceedings of SPIE Volume 6927 and is made available as an electronic reprint (preprint) with permission of SPIE. One print or electronic copy may be made for personal use only. Systematic or multiple reproduction, distribution to multiple locations via electronic or other means, duplication of any material in this paper for a fee or for commercial purposes, or modification of the content of the paper are prohibited. 


\title{
Ion-implanted compliant and patternable electrodes for miniaturized dielectric elastomer actuators
}

\author{
Samuel Rosset, Muhamed Niklaus, Venjamin Stojanov, Arnaud Felber, Philippe Dubois, and \\ Herbert R. Shea \\ École Polytechnique Fédérale de Lausanne, Switzerland
}

\begin{abstract}
This article presents metal ion implantation as an alternative technique to fabricate compliant electrodes for small-size dielectric elastomer actuators. When reducing the size of these actuators to below $1 \mathrm{~cm}$, the ability to pattern the electrodes is added to the need for compliance. Metal ion implantation on Polydimethylsiloxane (PDMS) layers allows the creation of conductive and compliant electrodes, which can be easily defined by photolithography or with a shadow mask. Mechanical testing show that implantation has a limited impact on the PDMS' properties, with a Young's modulus increase of 50\%-200\% depending on the dose. Uniaxial stretching tests show that conductivity is conserved for strains up to $50 \%$ and present no hysteresis. Dielectric breakdown tests were conducted for Au and Pd implantations, which exhibited high breakdown fields $(>100 \mathrm{~V} / \mu \mathrm{m})$, similar to non-implanted PDMS layers. Other advantages of ion implanted electrodes include transparency and a negligible mass. Buckling mode diaphragm actuators were fabricated with ion-implanted electrodes and exhibited out-of-plane displacements up to $7 \%$ of their lateral dimensions.
\end{abstract}

Keywords: Dielectric elastomer actuator, Metal ion implantation, Polydimethylsiloxane, Electrodes

\section{INTRODUCTION}

Dielectric Elastomer Actuators (DEAs) are a promising new kind of actuator combining large strains and large force. One of the key requirement of this technology is compliant electrodes: electrodes that can sustain large strains while remaining sufficiently conductive. Carbon powder, either mixed with grease or with the unpolymerized elastomer is the most popular and widespread method to create compliant electrodes for DEAs. ${ }^{1-4}$ It has the advantage of being a low cost method that does not require special equipment. However, this technology is hardly applicable to miniaturized DEA devices, for which it is necessary to pattern the compliant electrode, in order to produce many electrically-independent devices on a single substrate.

Several methods have been investigated and tested to make small patternable electrodes, such as carbonblack pattern-transfer with a Polydimethylsiloxane (PDMS) stamp, ${ }^{5}$ or thin-film deposition of a metallic layer (by sputtering or evaporation). The first solution has a limited resolution, and mechanical fixation of the carbon particles is problematic if no further layers are added to the device. The second solution has a high impact on the mechanical properties of the elastomer layer on which it is deposited and exhibit very small strains capabilities $(<3 \%)$. Pimpin and al. patterned an evaporated gold electrode in order to reduce the stiffening impact of the metal on the PDMS and to increase the deformation capability of the electrodes. ${ }^{6}$

To address some of the shortcomings of the methods mentioned above, we propose metal ion implantation as a way to create plain compliant electrodes, without forming a metallic layer. Filtered Cathodic Vacuum Arc (FCVA) is used as the implantation technique, as it can produce large ions currents at low energy, which allows implanting metallic particles at a density of $\sim 10^{22}$ at $/ \mathrm{cm}^{3}$ a few nanometers below the surface, and in a short amount of time $(2-5 \mathrm{~min}) .{ }^{7}$ The resulting electrodes exhibit low surface resistivity $(<100 \Omega /$ square $)$, good stretching capabilities, and can easily be patterned by using a lift-off process or a shadow mask. This paper reports on mechanical, electrical, and optical characterization of metal-implanted electrodes on PDMS.

Corresponding author: Samuel Rosset, EPFL-LMTS, rue Jaquet-Droz 1, cp 526, 2002 Neuchtel, Switzerland, samuel.rosset@a3.epfl.ch, tel +41327205437

Electroactive Polymer Actuators and Devices (EAPAD) 2008, edited by Yoseph Bar-Cohen

Proc. of SPIE Vol. 6927, 69270W, (2008) · 0277-786X/08/\$18 · doi: 10.1117/12.775727

Proc. of SPIE Vol. 6927 69270W-1 


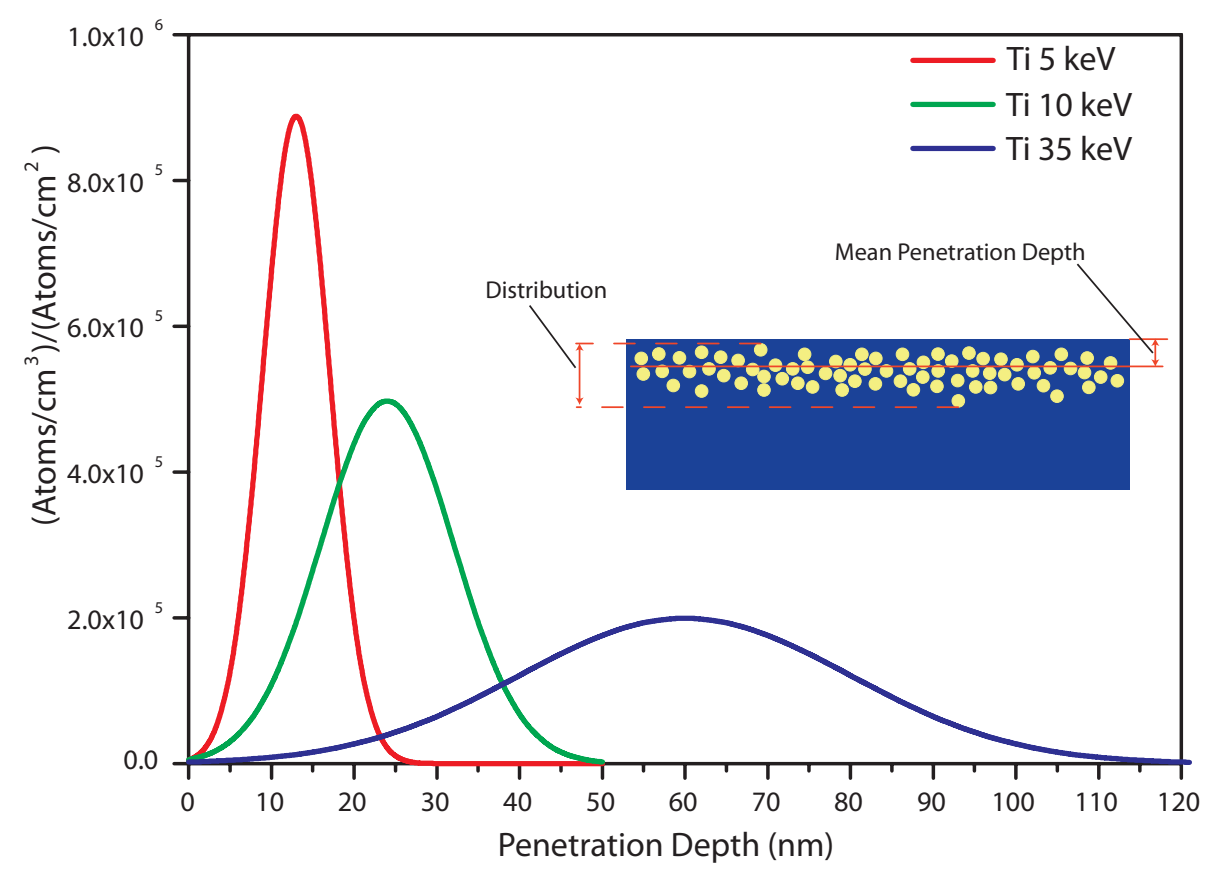

Figure 1. SRIM simulation of Ti Ion penetration depth versus ion energy. Implanting at low energy allows obtaining a dense distribution of metallic particles close to the surface.

\subsection{Implantation parameters}

The main parameters which characterize the implantation are:

1. The acceleration energy of the ions (eV), which, together with the atomic number $z$ of the implanted metal, determines the penetration depth (Fig. 1). Low accelerating energies are required to form a compact layer of implanted particles close to the surface. We use energies in the range of $2-5 \mathrm{keV}$ for our implantations.

2. The ion dose $\left(\mathrm{at} / \mathrm{cm}^{2}\right)$, which will determine the number of implanted ions, and therefore the final volumic density of metallic particles. This will have an impact on the electrical and mechanical properties of the implanted layer: the dose must be carefully chosen so that the implanted layer is sufficiently conductive while keeping the impact on the mechanical properties of the layer as small as possible. We use ion doses in the range of $10^{16}-10^{17}$ at $/ \mathrm{cm}^{2}$ for our implantations.

3. The ion current, which determines the time needed to implant a desired dose. This is a key parameter, as it is desirable to have short implantation times for practical and economic reasons. Conventional implanters such as those used for Si doping are not efficient at low energies and several hours to several days of implantation are needed to reach the targeted dose. This is the reason why we use FCVA as an implantation technique, for our system has an average current of $3 \mathrm{~mA}$ for an accelerating potential of $-2.5 \mathrm{kV}$, which allows to reach the targeted conductivity in about 5 minutes of implantation.

4. The implanted element. We have conducted implantation tests with $\mathrm{Ti}, \mathrm{Au}$ and $\mathrm{Pd}$. Ti-implanted layers suffer from oxidation and their resistivity increases with time. Au- and Pd-implanted layers do not suffer from this problem and exhibit very stable surface resistivity versus time.

\section{MECHANICAL AND ELECTRICAL PROPERTIES}

Electrodes for DEAs need to have the smallest possible impact on the Young's modulus of the PDMS on which they are created, while being sufficiently conductive. Surface resistivity and change of the Young's modulus were measured as a function of dose for Titanium and Gold implantations. The Young's modulus was measured by 

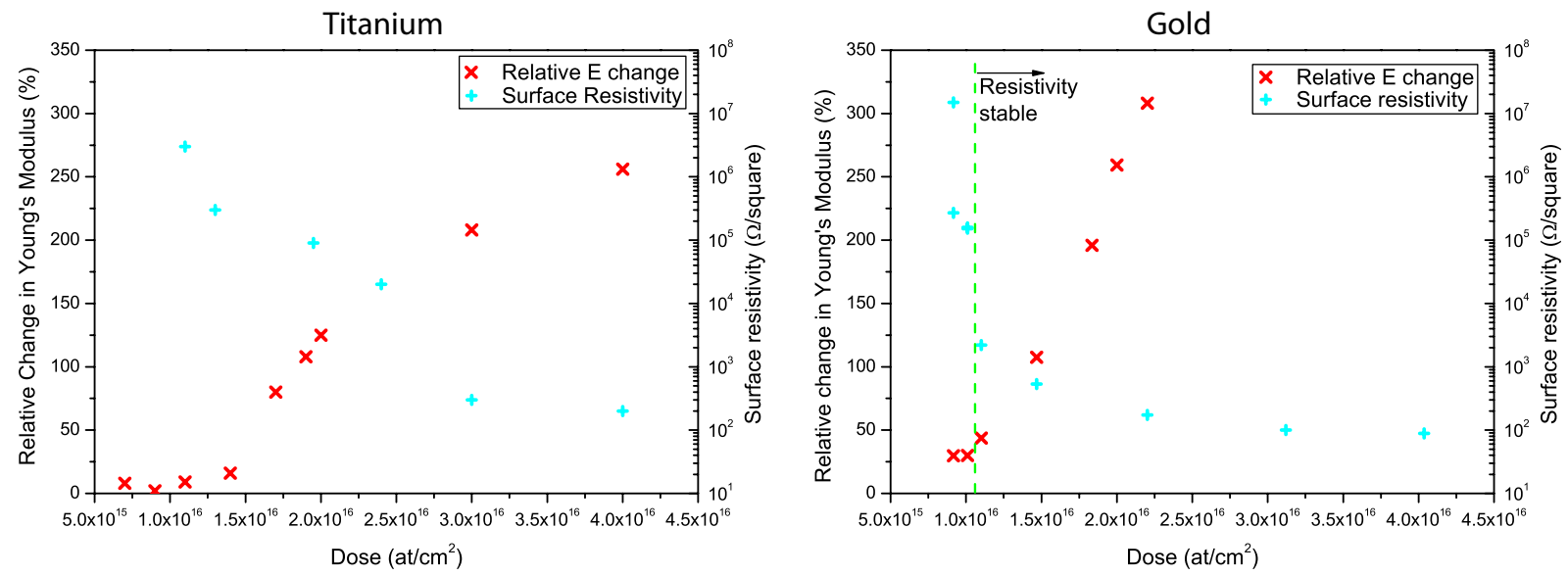

Figure 2. Relative change of Young's Modulus (E) and surface resistivity for FCVA implantation at $5 \mathrm{keV}$ of Ti (left) and $\mathrm{Au}$ (right) for different doses.
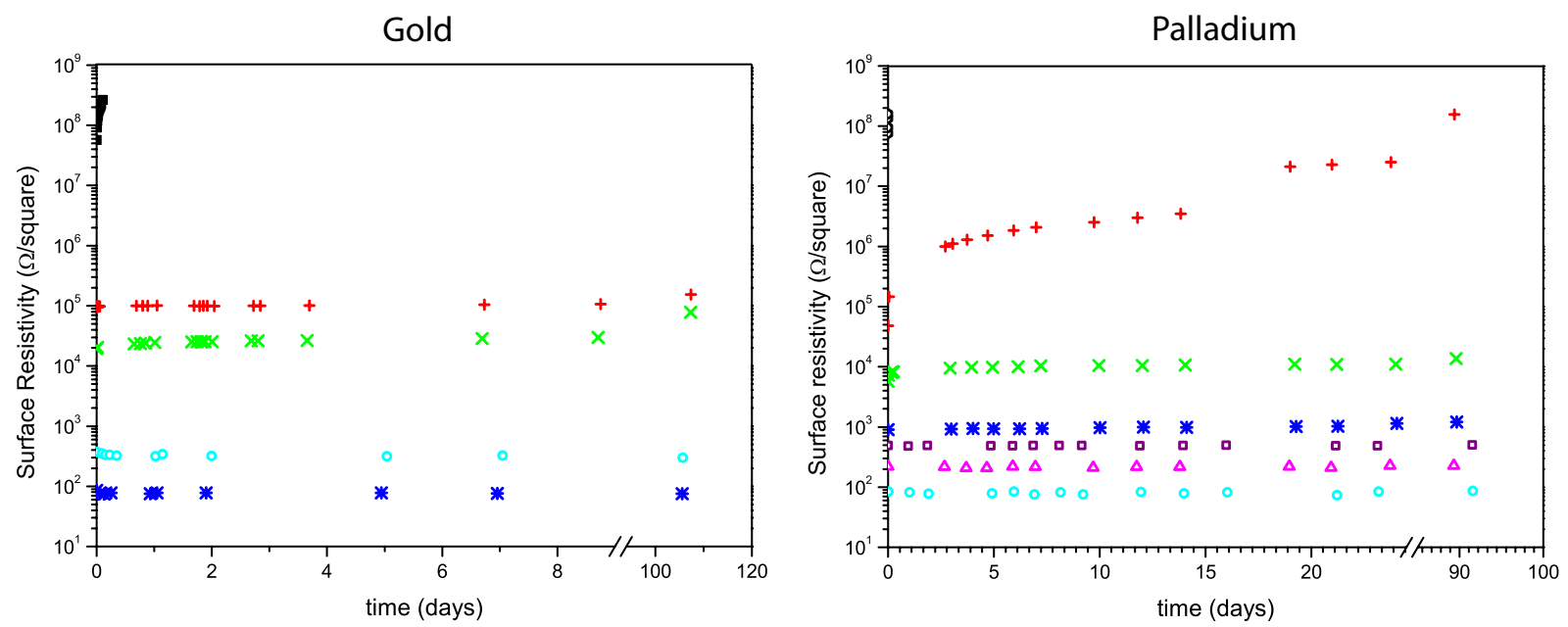

Figure 3. Surface resistivity vs. time for different $\mathrm{Au}$ (left) and Pd (right) doses. The different doses lead to different values of the surface resistivity. Implanted layers with an initial resistivity lower than $10 \mathrm{k} \Omega / \mathrm{square}$ do not show an increase of surface resistivity with time.

the bulge test technique ${ }^{8}$ on suspended $20 \times 20 \mathrm{~mm}^{2}$ square membranes made with a $20 \mu \mathrm{m}$-thick layer of Dow Corning Sylgard 186 PDMS. The Young's modulus was measured before and after implantation, and the relative change of Young's modulus was calculated (fig. 2).

\subsection{Resistance and Stability of Resistance}

Transmission electron microscopy shows that when the metallic ions penetrate the PDMS' surface, they tend to form clusters whose size depends on the dose. At low doses $\left(<10^{16} \mathrm{at} / \mathrm{cm}^{2}\right)$ the implanted particles' density is low and they are too far apart from each other to create a conduction path. Consequently, resistivity remains very high (above measurable range), and the impact on Young's modulus is low. For higher doses, a sharp decrease in resistivity is observed, which also coincides with a rapid increase of the stiffness of the membrane. This is the percolation threshold: the density of metallic particles becomes high enough so that conduction paths appear in the implanted layer. For $\mathrm{Au}$, the percolation region is particularly narrow: the resistivity drops from $>10^{9} \Omega$ /square to $100 \Omega$ /square for doses between $10^{16} \mathrm{at} / \mathrm{cm}^{2}$ and $1.5 \cdot 10^{16} \mathrm{at} / \mathrm{cm}^{2}$.

Stability of the resistivity is a concern for titanium layers, which suffer from oxidation: their resistivity quickly increases if left in ambient air. ${ }^{9}$ On the other hand, gold-implanted layers exhibit very stable resistivities vs. time for doses higher than $1.2 \cdot 10^{16}$ at $/ \mathrm{cm}^{2}$. Below this limit, resistivity increases with time, probably due to 

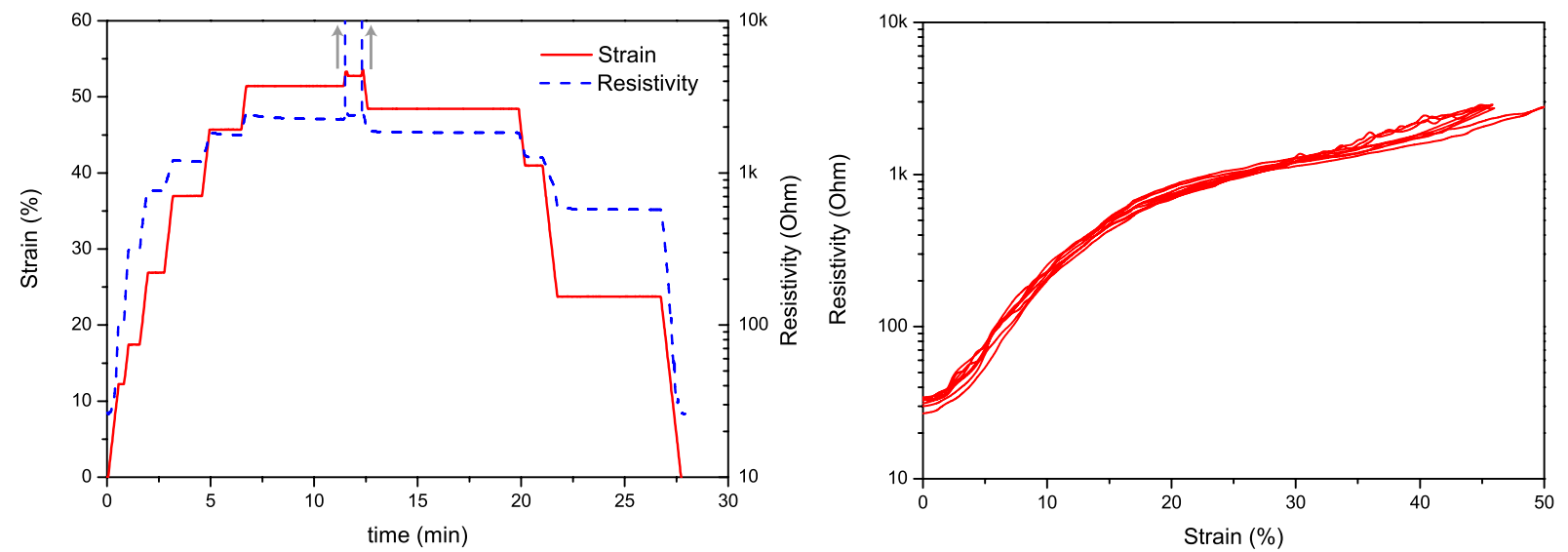

Figure 4. Left: Step measurement of electrical resistance and uniaxial strains between 0 and $55 \%$ of a PDMS stripe with an Au-implanted layer. Right: Cyclic strain-resistance measurement of a gold-implanted PDMS stripe.

diffusion. The same behavior is observed for Pd implantations; for both metals, implanted layers with an initial resistivity below $10 \mathrm{k} \Omega$ /square exhibit an excellent time stability (Fig. 3).

\subsection{Resistance Change for Large Strains}

One of the important characteristics that a compliant electrode must exhibit, is its ability to remain conductive during stretching. Through the use of bulge test measurements, we have shown that implantation had a low impact on the mechanical properties of freestanding membranes if the dose is correctly chosen, but we also need to quantify the resistivity variations of implanted layers during deformation.

Uniaxial tension tests were conducted on gold implanted samples that consisted of PDMS (Sylgard 186) stripes $(3 \mathrm{~mm} \times 8-18 \mathrm{~mm} \times 200 \mu \mathrm{m})$ that were fixed to a Schenk tension equipment. Both extremities of the tested stripe were metalized and connected to a Keithley 2000 multimeter to record its electrical resistance. The displacement signal from the tension machine was fed to another Keithley 2000 multimeter. Both instruments were connected to a PC that recorded the electrical resistance vs. displacement data. Two types of measurements were conducted:

- Single cycle with steps : The sample was deformed at a rate of $2 \mathrm{~mm} / \mathrm{min}$. At different points during the measurement, the motor was stopped and the deformation kept constant for a few seconds to test the resistance's stability. The stripe's stretching was increased up to the point where resistivity was higher than the multimeter's range, at which time the deformation's direction was changed and the tensile stress relaxed up to the point of zero deformation.

- Several continuous cycles : The sample was extended at a rate of $3 \mathrm{~mm} / \mathrm{min}$ from 0 strain up the the point where it looses conductivity, at which point the deformation's direction was changed and the sample was brought back to its initial size at the same rate. Several of such cycles were performed on the sample.

Samples with an electrode made by $\mathrm{Au}$ cathodic sputtering were also tested to compare standard metallic electrodes with metal ion implantated electrodes in terms of strain capabilities.

The step measurements show that resistivity remains stable while holding a position and that deformation up to $40 \%-45 \%$ can be achieved with stable conductivity. Resistivity increases from a initial value of $20-30 \Omega$, up to a few $\mathrm{k} \Omega$ at $45 \%$ strains, and becomes an open circuit for strains larger than $55 \%$. The samples had sputtered metallic gold contacts at both ends to improve electrical contact with the buried implanted layer, and it is therefore not perfectly clear if the strain limit before conductivity loss is due to the implantated region, or to cracks appearing in the sputtered contact area. Loss of conductivity is completely reversible when the strain is reduced (Fig 4 left). Cyclic measurements confirm that there is no hysteresis within a cycle, and that after several tension-relaxation cycles, the resistivity of the undeformed stripe is not altered (Fig 4 right). To suppress 


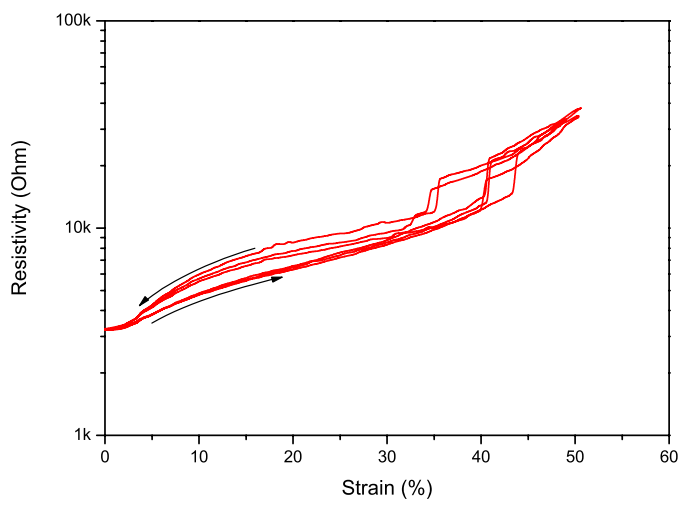

Figure 5. Cycle strain-resistance measurement of a gold-implanted layer without metallic contacts on the extremities.

the possible influence of the sputtered gold contact, similar samples were fabricated, but electrical contact was directly made to the implanted layer, without gold pads.

Without gold contacts, the initial resistivity of the undeformed sample is higher due to the difficulty to contact the buried implanted layer. Maximal deformation before loss of conductivity is slightly enhanced by the absence of gold contacts. Depending on the sample, it could go up to $60 \%$, but always above $50 \%$ strain, while remaining conductive. However, without gold pads, a small hysteresis was observed in the cycles, but the final resistivity was always the same than the initial unstrained resistivity (Fig. 5). Furthermore, some samples presented a resistivity step, at which point a rapid increase in resistivity was observed. It is not clear if the hysteresis and steps are due to the extension of the implanted electrode, or if this is caused by the rather poor contact with the buried metallic particles.

Sputtered samples were also fabricated to compare metal ion implantation's elongation capabilities with those of plain metallic electrodes. Sputtering was conducted in a Balzers SCD-030 bench top sputtering system for 3' at $60 \mathrm{~mA}$, in order to obtain samples with a conductivity comparable to that of the implanted samples $(20-30 \Omega$, $\approx 25 \mathrm{~nm}$ ). For strains greater than $1 \%$, sputtered samples exhibited cracks perpendicularly to the direction of deformation. If the strain is further increased, the cracks widen and conductivity is quickly lost (at strain levels between $2.5 \%$ and $3.5 \%$ ) (Fig. 6). When the strain is brought back to 0 , the cracks close, and initial conductivity is recovered. Compared to ion implanted electrodes, plain metallic electrodes can only be deformed of a few percent before loosing conductivity due to the formation of cracks. Consequently standard metallic electrodes are unsuitable for DEAs, for which deformations above $10 \%$ are desirable. One possible workaround that allows using sputtered layer as electrodes is to pattern them (for example in zig-zag). ${ }^{3,6}$

\section{DIELECTRIC BREAKDOWN}

Compared to carbon-based or sputtered electrodes, ion implanted electrodes will modify the surface of PDMS, due to ion penetration into the PDMS. Carbonization, chains scission and increase of surface roughness are likely to appear due to damage created by the dissipation of the incoming ions' energy. Concerns can be raised on the influence of ion implantation on the dielectric breakdown of the PDMS, as it is possible that the implantation-induced damage leads to a decrease of the dielectric strength of the PDMS. We have conducted extensive dielectric breakdown field measurements on different PDMS (Dow Corning Sylgard 186 and Nusil CF19-2186) of varying thicknesses $(19-35 \mu \mathrm{m})$. Samples were prepared in such a way that the capacitor zone in which dielectric breakdown occurs was free from mechanical deformation due to the application of the electrical contacts, that can have an influence on the measurement (Fig. 7). The implanted zone has one side on the top $\mathrm{Au}$ electrode, and the other side covering the bottom electrode, separated by the PDMS layer. This active zone is far from the electrical connections where mechanical stress which deform the PDMS is applied. Consequently, deformation due to electrical contacts will have no impact on the maximal voltage that can be applied to the 

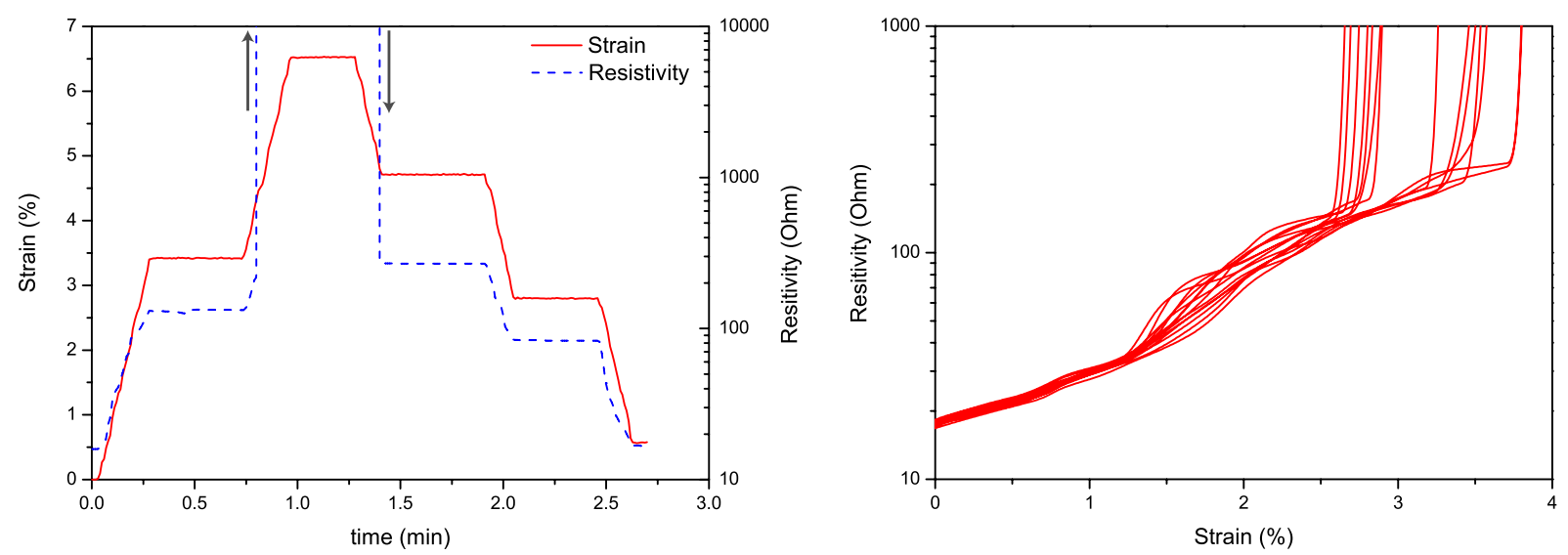

Figure 6. Left: Step measurement of electrical resistance for uniaxial strains of a PDMS stripe with an Au sputtered electrode. Right: Cycle strain-resistance measurement of the same stripe. Plain metallic electrodes exhibit very poor stretch capabilities.

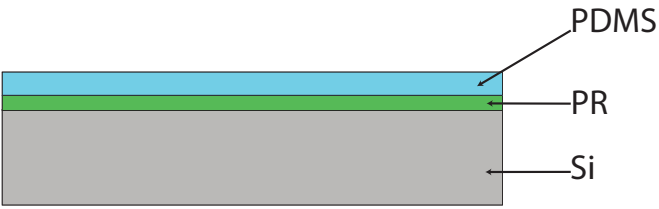

2

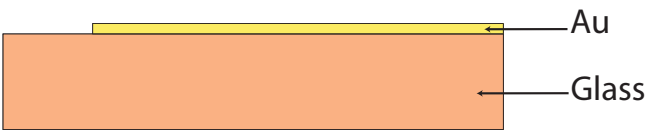

3

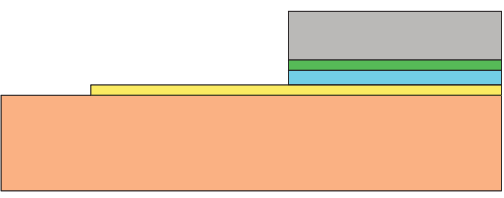

4

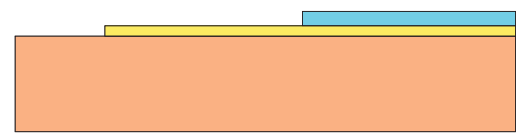

5

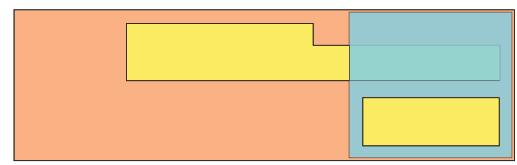

top view

6

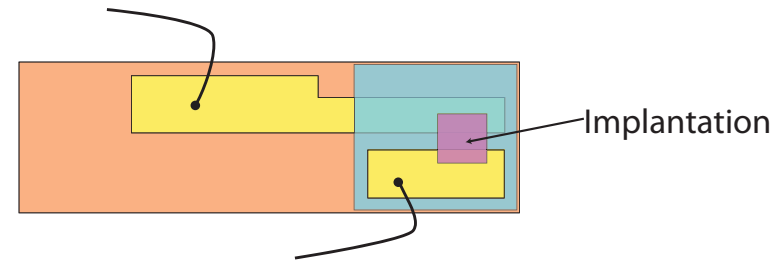

Figure 7. Process flow for the fabrication of the dielectric breakdown test chips. 1) A sacrificial layer of photoresist (PR) is spun on a Si wafer. A PDMS layer is applied on top of it by spin coating and cured $\left(100^{\circ} C\right.$ and RT). The wafer is then diced into $25 \times 25 \mathrm{~mm}^{2}$ chips. 2) A gold electrode is sputtered through a shadow mask on a microscope glass slide. 3) A Si-PR-PDMS chip is bonded on the glass slide after an oxygen plasma treatment. 4) The assembly is dipped into acetone to dissolve the PR layer and remove the Si transfer chip. 5) A top gold electrode is sputtered on the PDMS through a shadow mask. 6) Implantation is conducted through a shadow mask. Electrical contacts are made to the sputtered electrodes

structure. Implantations were conducted at $5 \mathrm{keV}$ for both $\mathrm{Au}$ and $\mathrm{Pd}$ samples. For Au, the implanted dose was $3.8 \cdot 10^{16} \mathrm{at} / \mathrm{cm}^{2}$. For Pd, no RBS calibration has yet been made, but the surface resistivity of the implanted layer was $\sim 100 \Omega$ /square, which is the same value than that of the gold implantations.

Values of breakdown voltage versus PDMS thickness are reported in figure 8 for Au-implantations, and in figure 9 for $\mathrm{Pd}$-implantations, with a linear fit to estimate the breakdown E field. Some datapoints with low breakdown voltages were discarded from the E field evaluation, as electrical failure for those samples was probably due to defects in the PDMS, such as dust or air bubbles. The local reduction in thickness at the electrode's periphery due to the applied field (and hence electrostatic force) was not taken into account, because it was measured with an optical profiler to be a fraction of a micrometer, which is much less than the thickness nonuniformity due to the spin-coating process $( \pm 1 \mu \mathrm{m})$. To compare the dielectric strength of implanted PDMS 

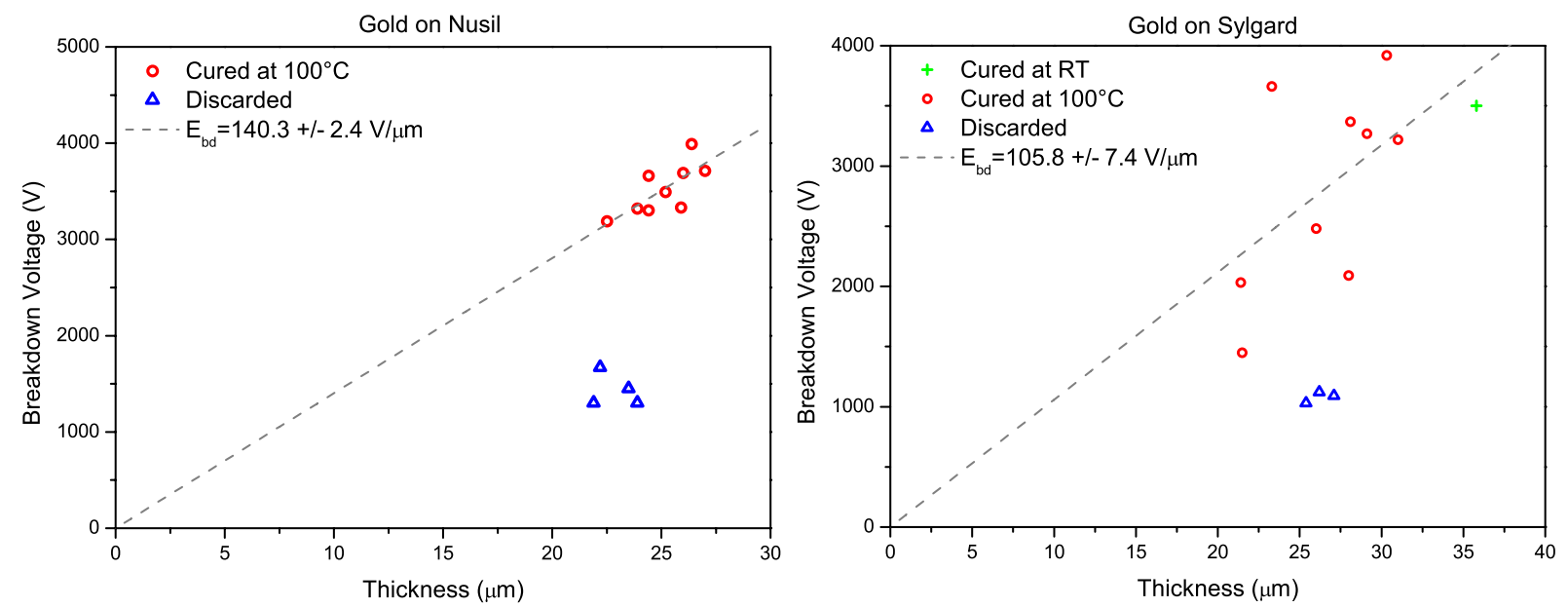

Figure 8. Breakdown voltage of Au-implanted Nusil (left) and Sylgard (right) layers at 5 keV, for different PDMS thicknesses.
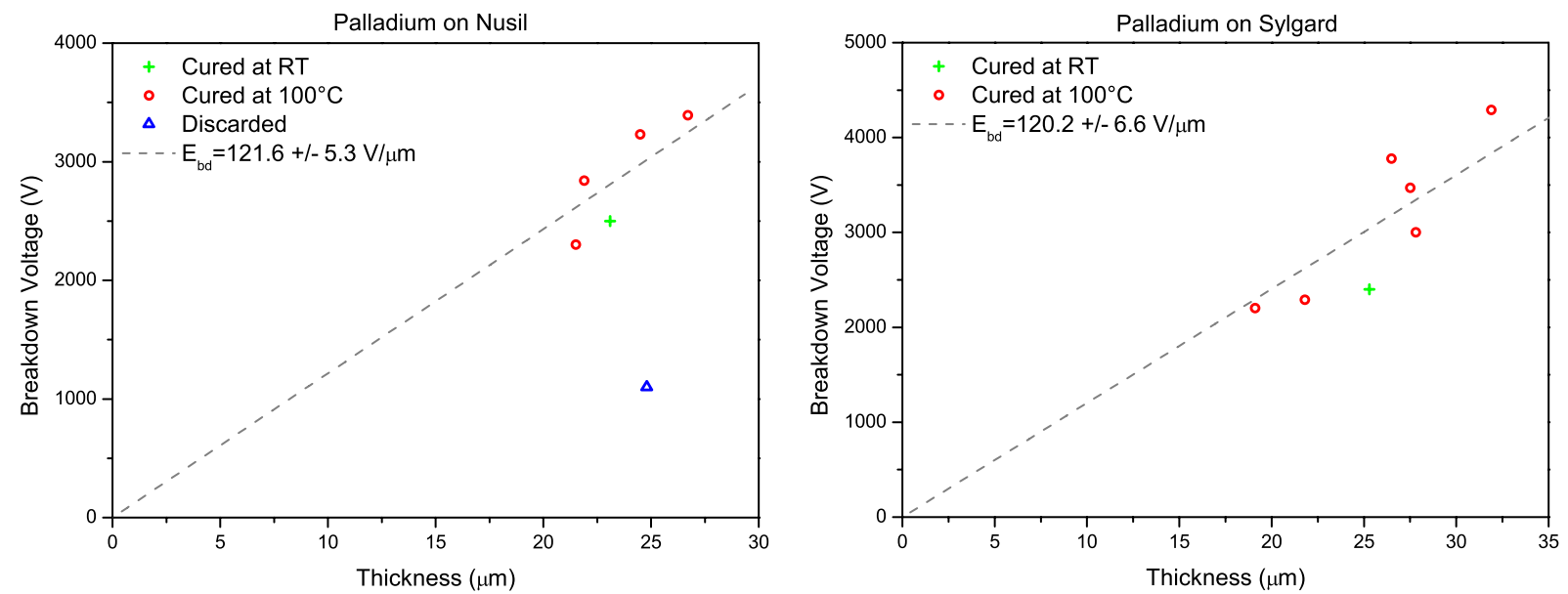

Figure 9. Breakdown voltage of Pd-implanted Nusil (left) and Sylgard (right) layers at 5 keV, for different PDMS thicknesses.
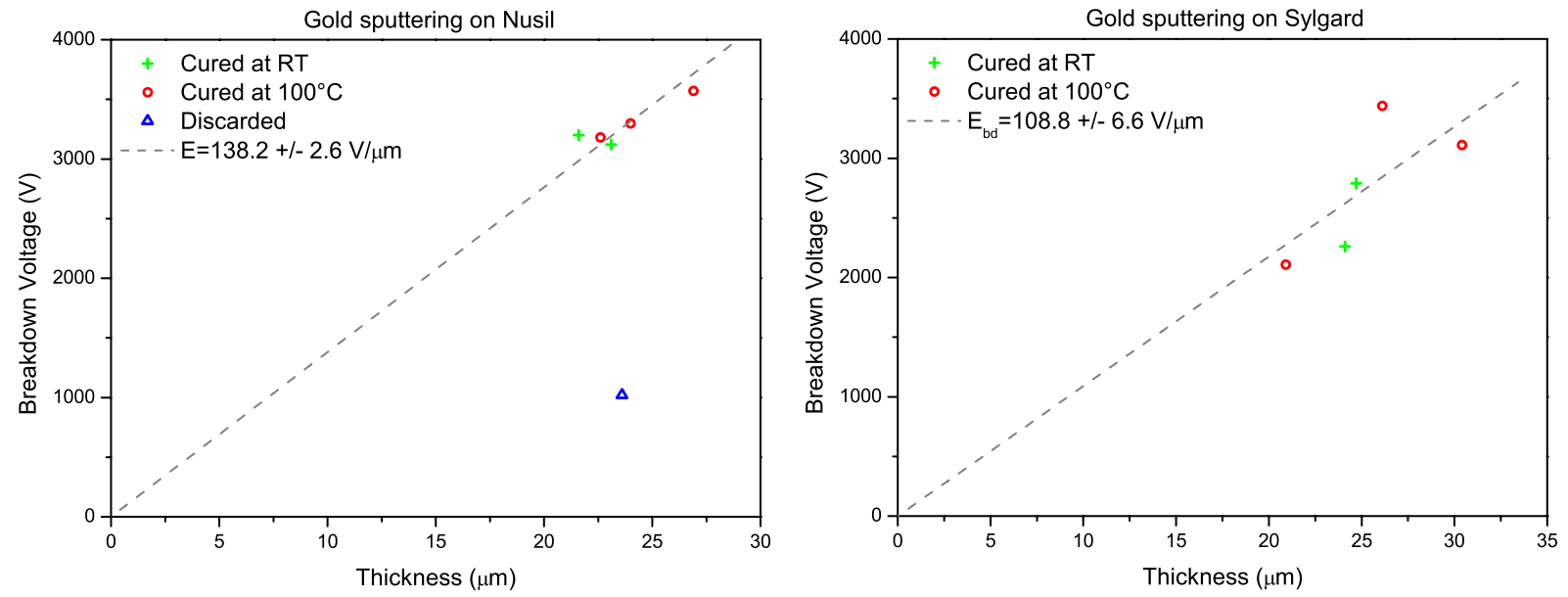

Figure 10. Breakdown voltage of Gold-sputtered Nusil (left) and Sylgard (right) layers, for different PDMS thicknesses. 
Table 1. Dielectric breakdown fields measured for two different PDMS (Sylgard 186 and Nusil CF19-2186) for different conditions: Not implanted, Pd- and Au-implanted

\begin{tabular}{c|c|c} 
& Sylgard $186(\mathrm{~V} / \mu \mathrm{m})$ & Nusil CF 19-2186 $(\mathrm{V} / \mu \mathrm{m})$ \\
\hline PDMS only & $108.8 \pm 6.6$ & $138.2 \pm 2.6$ \\
\hline Pd implantation & $120.2 \pm 6.6$ & $121.6 \pm 5.2$ \\
\hline Au implantation & $105.8 \pm 7.4$ & $140.3 \pm 2.4$ \\
\hline
\end{tabular}

to that of the pristine material, similar samples were prepared, but the implanted electrode was replaced by a gold-sputtered electrode. Because sputtering creates a metallic layer on top of the PDMS (as opposed to implantation, which creates an electrode inside it), the breakdown field measured between the sputtered top and bottom electrode is taken to be the breakdown field of the tested pristine PDMS (Fig. 10). Table 1 summarizes the obtained result for dielectric breakdown measurements.

The measurements show that ion metal implantation does not alter the dielectric strength of the PDMS on which it is conducted, at least not at the low energies involved here $(5 \mathrm{keV})$. Indeed, the breakdown fields obtained for implantation (for $\mathrm{Au}$ as well as Pd) are very close to those of unimplanted PDMS, and even superior in some cases, which shows that the damages provoked by the incoming ions are not the dominant factor in PDMS dielectric breakdown. Ion implantation can therefore be used to make compliant electrodes for DEAs without fear of altering the elastomer's dielectric strength. One can also observe that measurements made on Sylgard have more scatter than those made on Nusil. Nusil CF19-2186 is also easier to spin-coat than Sylgard 186 and leads to more uniform thickness on the wafer surface. This can be observed on the measurements by the wider range of thicknesses obtained with Sylgard, although the same rotation speeds were used for the 2 different PDMS: from a reproducibility standpoint, Nusil CF19-2186 appears to be more suitable.

Although we have seen in a previous study that the curing temperature of the PDMS has an important influence on the mechanical parameters of the resulting cured PDMS layer (Young's modulus and residual stress), ${ }^{9}$ it does not have any impact on the dielectric strength of the PDMS, as the datapoints from layers cured at room temperature lie on the same line as data from the layers cured at $100^{\circ} \mathrm{C}$ (Fig. 8-10). Consequently, curing temperature can be selected in order to tune the membranes mechanical properties for a given application, without fear of an influence of the dielectric strength of the structure.

The measured dielectric strength of both PDMS is much higher than the value given by the manufacturer (13 V/ $/ \mu \mathrm{m}$ for Sylgard 186 and $28.7 \mathrm{~V} / \mu \mathrm{m}$ for Nusil CF19-2186. The differences can come from a security margin given by the manufacturer. Additionally, dielectric strength of thin-film may be higher than that of the bulk material due to the alignment of the polymer strings. Other authors also report on high breakdown field for those two polymers (as well as other). ${ }^{3}$

\section{APPLication to ACTUATORS}

We have applied our technology to the fabrication of diaphragm DEAs with ion implanted electrodes. Thin circular PDMS membranes $(2-3 \mathrm{~mm}$ in diameter, $20-30 \mu \mathrm{m}$ thick) were fabricated and implanted on both sides with gold ions. Upon application of a voltage between the two electrodes, out of plane deflection is produced by the area expansion (Fig. 11). Characterization of the actuator under mechanical load was done by applying a distributed force (air pressure) on one side of the membrane. The vertical deflection of the membrane's center was measured for different values of voltage and pressure. Experimental data were found to be in excellent agreement with an analytical model ${ }^{7}$ (Fig 12). Voltage-induced deflection up to $7 \%$ of the actuator's lateral dimension are obtained for this sample.

\section{CONCLUSIONS}

Ion implanted electrodes are well suited for small size DEAs, for which patterning of the electrodes in complex shapes is desirable. In this paper, we have shown that metal ion implantation meets the main criteria for application to DEAs: time stability of resistivity, strain capabilities higher than $40 \%$, no impact on the dielectric 

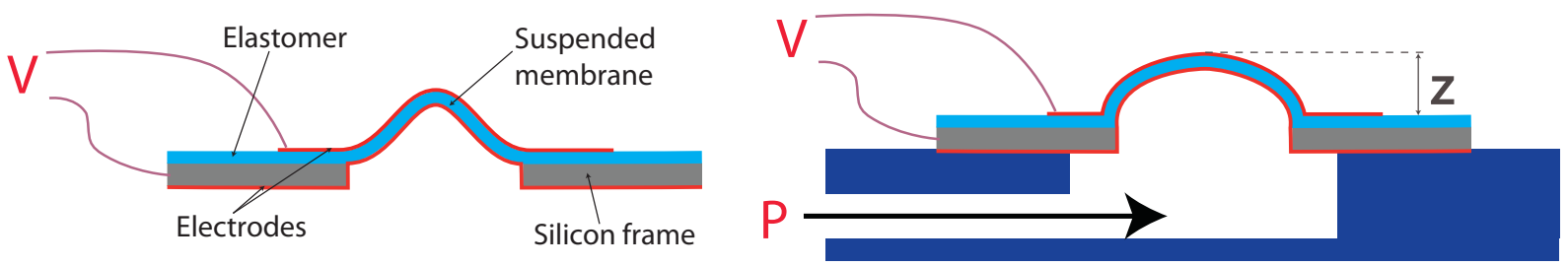

Figure 11. Working principle of a DEA membrane actuator. Left: Without any external force, the membrane buckles when a sufficiently high voltage is applied between the electrodes. Right: to test the actuator under a mechanical load, it was mounted in an airtight socket. A pressure difference is applied between the two side of the membrane. Applying a voltage modulates the membrane's deformation.
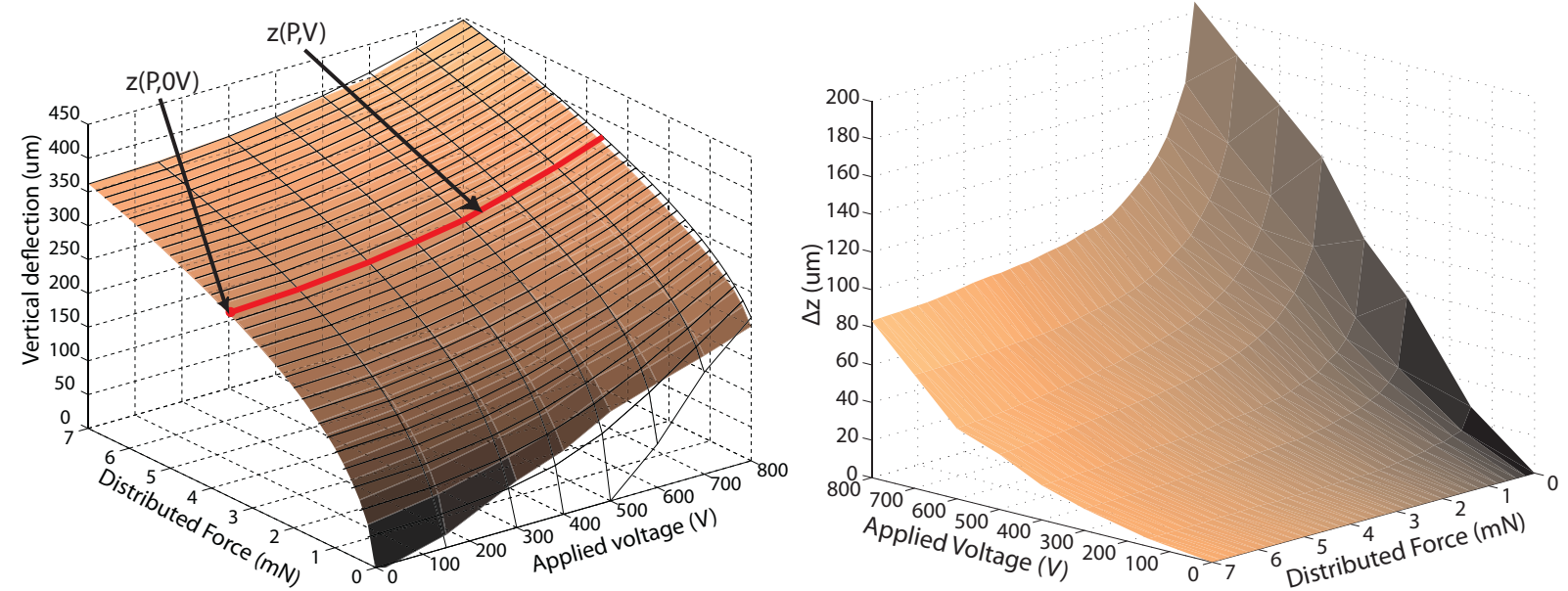

Figure 12. Vertical deflection of a 3-mm membrane $(t=22.5 \mu \mathrm{m})$ as a function of applied voltage and distributed force (left). Wireframe: theoretical displacement obtained with the model. Colored plane: measured data. Right: measured voltage-induced deflection $(\Delta z)$ obtained by removing the pressure contribution to the vertical motion: $\Delta z(P, V)=$ $z(P, V)-z(P, 0 V)$.

strength of the PDMS... There are other important characteristics of electrodes made by metal ion implantation that we shall mention:

Advantages of ion implantation: Implanted electrodes are only a few tens nanometers thick and buried inside the PDMS, making them particularly suitable to build stacked structures, in order to reduce the actuation voltage: with implanted electrodes, there isn't a large inactive volume caused by the electrodes. Implanted electrodes also have a negligible mass, which allows a higher energy density to be stored in the actuator. These two advantages are also shared with evaporated/sputtered thin metallic electrodes, but these have an important impact on the mechanical's properties of the PDMS, unless carefully patterned. ${ }^{6}$ Compared to metallic electrode, or to carbon grease, implanted electrodes also have a very strong adhesion to the PDMS' surface, as the metallic particles are located inside the PDMS matrix. Unlike carbon-based electrodes, ion-implanted electrodes are clean to fabricate and do not involve the manipulation of potentially hazardous materials such as nanoparticles.

Drawbacks of ion implantation: Metal ion implantation unfortunately requires equipment, that (unlike for evaporated or sputtered electrodes) is not commonly found in every clean room. We built our custom experimental FCVA setup, with a $1 \mathrm{~cm}^{2}$ beam, and which is coupled to a $\mathrm{x}-\mathrm{y}$ stage for larger area scanning. Although ion implanted electrode could be applicable to larger (macroscale) devices, lack of readily available equipment is a large inconvenient to the widespread use of this technology. Some commercial FCVA implanters, mainly designed and optimized to deposit hard coatings on cutting tools, do exist and could be applicable to treat large PDMS areas, provided their ion current can be set low enough that it won't burn the target and that the desired dose can be easily controlled.

Ion implanted electrodes have been successfully applied to working diaphragm DEAs, which exhibited outof-plane displacements of about $7 \%$ of their lateral size. These electrodes offer an interesting alternative to 
mainstream methods and have application beyond the field of electroactive polymers, such as producing highly adhesive stretchable electrodes on polymers.

\section{ACKNOWLEDGMENTS}

We wish to thank the COMLAB staff for help with device fabrication, and the service of micro and nanoscopy of the University of Neuchâtel for the access to the tension equipment. We also acknowledge financial support from the Swiss National Science Foundation grant \#20021-111841, and from the EPFL.

\section{REFERENCES}

[1] Carpi, F., Chiarelli, P., Mazzoldi, A., and De Rossi, D., "Electromechanical characterisation of dielectric elastomer planar actuators: comparative evaluation of different electrode materials and different counterloads," Sensors and Actuators A: Physical 107(1), 85-95 (2003).

[2] Pelrine, R., Kornbluh, R., Pei, Q., and Joseph, J., "High-speed electrically actuated elastomers with strain greater than 100\%," Science 287(5454), 836-839 (2000).

[3] Pelrine, R., Kornbluh, R., Joseph, J., Heydt, R., Pei, Q., and Chiba, S., "High-field deformation of elastomeric dielectrics for actuators," Materials Science and Engineering: C 11(2), 89-100 (2000).

[4] Heydt, R., Kornbluh, R., Pelrine, R., and Mason, V., "Design and performance of an electrostrictive-polymerfilm acoustic actuator," Journal of Sound and Vibration 215(2), 297-311 (1998).

[5] Aschwanden, M. and Stemmer, A., "Low voltage, highly tunable diffraction grating based on dielectric elastomer actuators," in [Electroactive Polymer Actuators and Devices (EAPAD) 2007], 6524, 65241N-10, SPIE, San Diego, California, USA (2007).

[6] Pimpin, A., Suzuki, Y., and Kasagi, N., "Microelectrostrictive actuator with large out-of-plane deformation for flow-control application," Microelectromechanical Systems, Journal of 16(3), 753-764 (2007).

[7] Rosset, S., Niklaus, M., Dubois, P., and Shea, H. R., "Mechanical characterization of a dielectric elastomer microactuator with ion-implanted electrodes," Sensors and Actuators A: Physical In Press, Accepted Manuscript.

[8] Vlassak, J. J. and Nix, W. D., "A new bulge test technique for the determination of young's modulus and poisson's ratio of thin films," Journal of Materials Research 7(12), 3242-3249 (1992).

[9] Rosset, S., Niklaus, M., Dubois, P., Dadras, M., and Shea, H. R., "Mechanical properties of electroactive polymer microactuators with ion-implanted electrodes," in [Electroactive Polymer Actuators and Devices (EAPAD) 2007], 6524, 652410-11, SPIE, San Diego, California, USA (2007). 